Pacific Journal of Mathematics

A GENERALIZATION OF THE CHINESE REMAINDER

B. ARA II 


\title{
A GENERALIZATION OF THE CHINESE REMAINDER THEOREM
}

\author{
B. Arazi
}

Let $X$ be a set of $r$ nonnegative integers, and let $B_{t}$, $i=1,2,3, \cdots, t$ be the unordered sets of residues of the elements of $X$ modulo $m_{i}$, where it is not known which element in $X$ produces a given element in $B_{l}$.

For the case where $r=1$, the Chinese Remainder Theorem introduces necessary and sufficient conditions on the values of $m_{\text {t }}$ in order that $X$ may have a unique solution $\bmod \Pi_{t=1}^{t} m_{\text {• }}$.

This paper introduces such conditions for the case where $r \geqq 1$.

Introduction. The Chinese Remainder Theorem states that the system of congruences $x \equiv b_{i}\left(\bmod m_{l}\right), i=1,2,3, \cdots, t$ has a unique solution $\bmod \prod_{i=1}^{t} m_{l}$, iff $\left(m_{i}, m_{j}\right)=1$ for $i \neq j$.

This leads to the folloing question: Let $X=\left\{X_{1}, X_{2}, \cdots, X_{r}\right\}$ be a set of nonnegative integers (not necessarily distinct) and let $B_{i}, i=$ $1,2,3, \cdots, t$, be the sets of residues of the elements of $X$ modulo $m_{i}$, where it is not known which element in $X$ produces a given element in $B_{l}$. If $0 \leqq X_{i}<\prod_{i=1}^{t} m_{\imath}$ for $1 \leqq j \leqq r$, and $\left(m_{l}, m_{\jmath}\right)=1$ for $i \neq j$, is it possible to determine the elements of $X$ uniquely, knowing the $B_{\imath}$ 's?

If a certain value $C$ appears in $X$ for $n$ times then $C(\bmod i)$ appears $n$ times in $B_{r}$. If there is only one value which appears in $X$ for $n$ times then in view of the Chinese Remainder Theorem it is possible to determine it uniquely and from it, the whole set $X$. This paper will therefore treat the most general case where every value appears for the same number of times and without loss of generality it can be assumed that all the elements of $X$ are distinct.

Before any attempt is made at answering the question which was posed, there are two facts which have to be taken into account.

(a) If for some $m_{i}, X_{i} \equiv X_{1}\left(\bmod m_{i}\right)$ then there is no sufficient information for determining $X_{i}$ and $X$ uniquely.

(b) If for some $m_{i}$, the set $X$ contains $m_{t}$ successive integers, which are the only elements in the set, then the set $B_{i}$ contains all integers from 0 to $m_{i}-1$, and $X$ cannot be determined uniquely.

Let $X_{r}$ and $X_{1}$ be the largest and smallest elements of $X$ respectively, and let $m_{1}<m_{i}$ for $i>1$. In order to take into account the two above-mentioned facts when finding an answer to our question, it is enough to require that $X_{r}-X_{1}<m_{1}$ and that the number of distinct 
elements in $X$ will not exceed $m_{1}-1$. With these restrictions, the answer to our question is negative ( $X$ cannot be determined uniquely). This can be demonstrated as follows:

Let $X_{r}>X_{r-1}>\cdots>X_{1}$. Let $p$ be a divisor of some $m_{k}, 1 \leqq k \leqq t$, and let $X_{l}-X_{\imath-1}=p$ for $i=2,3, \cdots, r$. Let $s \triangleq \Pi_{j \neq k} m_{\jmath}$, and let $q=$ $s \cdot p$. It can be shown that the set $Y=\left\{X_{1}+q, X_{2}+q, \cdots, X_{r}+q\right\}$ and the set $X$ have the same $B_{i}, i=1,2, \cdots, t$.

In fact, it is always possible to construct such a set $Y$, if $X$ has a periodic structure with periodicity $p$.

A necessary and sufficient condition that there should be no periodic set $Y$, is that $\left(r, m_{i}\right)=1$ for $i=1,2,3, \cdots, t$. (In the original form of the Chinese Remainder Theorem this condition is always fulfilled, since $r=1$.)

Interestingly enough, even this condition is not sufficient to determine $X$ uniquely. The following demonstrates a case where two different sets $X$ and $Y$ have the same residue sets $B_{1}$ and $B_{2}(t=2)$ although $m_{1}$ and $m_{2}$ are both primes.

$$
\begin{aligned}
X & =\{11,12,14,15,19,20\} \\
Y & =\{58,59,63,64,66,67\} \\
m_{1} & =11, m_{2}=13 . \quad\left(r=6<m_{1}<m_{2}\right) \\
B_{1} & =\{1,3,4,8,9,11\} \\
B_{2} & =\{1,2,6,7,11,12\} .
\end{aligned}
$$

This paper shows which are the conditions imposed on the values of $m_{i}$ and $r$, under which $X$ is determined uniquely.

\section{THEORY.}

Theorem. Let $X=\left\{X_{1}, X_{2}, \cdots, X_{r}\right\}$ be a set of distinct nonnegative integers. Let $X_{r}$ and $X_{1}$ be the largest and smallest elements of $X$ respectively, and let $m_{1}$ be an integer such that $r \leqq X_{r}-X_{1}<m_{1}$.

Let $m_{k}, k=2,3, \cdots, t$ be integers such that $m_{t}>m_{t-1}>\cdots>m_{2}>m_{1}$ and $\left(m_{i}, m_{j}\right)=1$ for $i \neq j$, and let $X_{r}<\prod_{i=1}^{t} m_{i}$.

Let $B_{i}$ be the sets of residues of the elements of $X$ modulo $m_{i}$ for $i=1,2,3, \cdots, t$, where it is not known which element in $X$ produces a given element in $B$. Let $s_{i j} \triangleq m_{i}-m_{j}$.

The set $X$ can be determined uniquely knowing the residue sets $B_{i}$ iff $\left(r, m_{i}\right)=1$ and $\left(r, m_{i}-n \cdot s_{i j}\right)<n+1$ for $n=1,2, \cdots, h-1$ where $h$ is the smallest integer such that $r>m_{\imath}-h \cdot s_{i j}$. This applies to $i=$ $1,2,3, \cdots, t-1$, and all $j<i$. 
Before proving the theorem it is worth while showing some corollaries.

COROLlaRY 1. If $r$ is a power of 2, a sufficient condition that $X$ may be determined uniquely is that $m_{\imath}$ should be odd for $i=1,2,3, \cdots, t$.

Corollary 2. $X$ can be determined uniquely if $\left(r, m_{i}\right)=1$ for $i=1,2,3, \cdots, t$, and $m_{i}>2 m_{t-1}$ for $i=2,3, \cdots, t$.

COROllary 3. X can be determined uniquely for all possible values of $r$ (as long as $r<m_{1}<m_{t}, i=2,3, \cdots, t$ ) if $m_{t}, i=1,2,3, \cdots, t$ are all primes and $m_{l}>2 m_{\imath-1}, i=2,3, \cdots, t$.

Proof. Notations: (1) The number of elements in a sequence $A$ is denoted by $l(A)$.

(2) Let $A$ and $B$ be sequences of numbers. The sequence $A$ is a 'sub-sequence' of $B$ iff $A$ consists of $l(A)$ elements which appear successively in $B$.

Step 1. Interpretation of the theorem in terms of cyclic shifts of binary sequences.

One way of interpreting the Chinese Remainder Theorem for the case where only two congruences are considered $(t=2)$, is as follows. Let $P$ and $Q$ be binary sequences with only one element of value 1 , which is also the first element in both sequences. Let $p \stackrel{\Delta}{=} l(P)$ and $q \stackrel{\Delta}{=} l(Q)$ (this definition of $p$ and $q$ holds also for the rest of the text) where $(p, q)=1$. Let $P$ and $Q$ be shifted cyclically through $k$ places until the sequences $P^{\prime}$ and $Q^{\prime}$ respectively are obtained, where the first element of both $P^{\prime}$ and $Q^{\prime}$ is 1 . Then $k=n \cdot p \cdot q$ for some integer $n$.

In the same way, the following lemma is an interpretation of the proposed theorem for the case $t=2$.

LEMMA 1. Let $P$ and $Q$ be binary sequences with $r$ elements of value 1 , where $q>p>r$ and $(p, q)=1$. The first $p$ elements of $Q$ are identical with the elements of $P$ whose first element is $1 . \quad$ Let $P$ and $Q$ be shifted cyclically through $k$ places until the sequences $P^{\prime}$ and $Q^{\prime}$ respectively are obtained such that the first $p$ elements of $Q^{\prime}$ are identical with the elements of $P^{\prime}$ and this has 1 as the first element. Then $k$ is only of the form $m \cdot p \cdot q$ for some integer $m$ iff $(r, p)=1$ and $(r, p-n(q-p))<n+1$ for $n=1,2, \cdots, h-1$ where $h$ is the smallest integer such that $r>$ $p-h(q-p)$.

Steps 2 to 7 of this proof deal with the proof of Lemma 1, and the general theorem will be proved only at Step 8 . 
Step 2. The case of a periodic $P$. If $P$ has a periodic structure, i.e. it consists of repetition of a sub-sequence $A$, where $l(A)=k<p$, then $P$ can repeat itself after $m \cdot k$ shifts, where $0<m \cdot k<p$, and $m$ is an integer. Since $P$ contains at least one element of value 0 (this follows from the fact that $p>r$ ) it follows that $k \geqq 2$.

If $s \stackrel{\Delta}{=} p / k(s$ is the number of sequences $A)$ then $s$ must be a divisor of $r$ (since $r$ elements of value 1 must be equally shared among all sub-sequences), which means that $(p, r)>1$. It follows that the condition stated in Lemma 1 is sufficient for $P$ to be nonperiodic.

Step 3. The case of a periodic $Q$. By applying to $Q$ the considerations applied above to $P$, it can be shown that the periodicity of $Q$ implies that $(q, r)>1$, where $Q$ consists of $s$ sub-sequences $A$, with $l(A)=k$. Let $b$ denote the number of elements of value 1 in $A$, then this means that $A$ contains $k-b$ elements of value 0 . On the other hand, $Q$ has at least $q-p$ elements of value 0 , which appear successively at its end (this follows from the definition of $Q$ in Lemma 1).

Postulate. If $(r, p)=1$ and $(r, p-n(q-p))<n+1$ for $n=$ $1,2, \cdots, h-1$ then $Q$ is nonperiodic. ( $h$ was defined in Lemma 1$)$.

Proof. Assume that $Q$ is periodic. Then in view of the preceding discussion, $q=s \cdot k$ and $r=s \cdot b$.

Let $d=q-p$. Since $(p, r)=1$, this means that $(q-d, r)=1$ and it follows that $(d, s)=1$.

It follows that one of the elements of the arithmetic progression $p, p-d, p-2 d, \cdots, p-(s-1) d$ is divisible by $s$. Let this element be denoted by $a_{n}$. It follows that $\left(r, a_{n}\right) \geqq s$. In order to show that the conditions $(r, p)=1$ and $(r, p-n(q-p))<n+1$ (for $n=1,2, \cdots, h-1)$ are sufficient for $Q$ to be nonperiodic it should be shown that $s-1 \leqq$ $h-1$. Let $a_{1}=p-(h-1) d$.

$$
q=a_{1}+h d=a_{1}-d+(h+1) d
$$

but $a_{1}-d<r$ (follows from the definition of $h$ ).

$$
\Rightarrow q<r+(h+1) d
$$

but $q=s \cdot k, r=s \cdot b$

$$
\Rightarrow s(k-b)<(h+1) d=(h+1)(q-p) .
$$

It was shown before that the sub-sequence $A$ contains $k-b$ elements of value 0 , where the minimum number of these elements is $q-p$. Since $k-b \geqq q-p$ it follows that $s<h+1$, which means that $s-1 \leqq h-1$. 
This completes the proof of the postulate and it can be concluded that the conditions stated in Lemma 1 are sufficient for $Q$ to be nonperiodic.

However, even for nonperiodic $P$ and $Q$, it is possible to find the sequences $P^{\prime}$ and $Q^{\prime}$ described in Lemma 1 where $0<k<p \cdot q$, as shown by the following example.

$P=1100001, Q=11000010000(p=7, q=11)$. If both $P$ and $Q$ are shifted cyclically to the left for 50 places, the following sequences are obtained, $P^{\prime}=1000011, Q^{\prime}=10000110000$.

Step 4. Analysing $P, P^{\prime}, Q$ and $Q^{\prime}$ assuming their existence for $0<k<p \cdot q$. Let it be assumed that the sequences $P, P^{\prime}, Q$ and $Q^{\prime}$ introduced in Lemma 1 exist for $0<k<p \cdot q$. This Step and the following one will show that this assumption is not valid under the conditions introduced later in the Lemma.

Let $i \equiv k(\bmod p)$ and $j \equiv k(\bmod q)$. It is clear that $i \neq 0$ and $j \neq 0$, otherwise $P=P^{\prime}$ or $Q=Q^{\prime}$. The case where $i=j$ is analysed at this Step.

In order that $Q^{\prime}$ may be obtained from $Q$ by a cyclic shift, $Q$ must have somewhere in it $q-p$ successive zeros (which are transferred to its end by the cyclic shift that produces $Q^{\prime}$ ). These zeros are followed by a 1 (which is transferred to the beginning of $Q^{\prime}$ ) and therefore they cannot be part of the last $q-p$ zeros at the end of $Q$. It follows that $Q$ has in it $q-p$ successive zeros confined to the first $p$ places, which consist of the sequence $P$.

The sequences $Q, P, Q^{\prime}$ and $P^{\prime}$ thus have the following form.
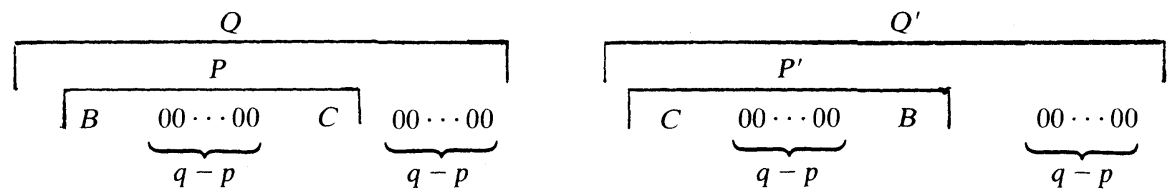

FIGURE 1

where $B$ and $C$ are sequences starting with a 1 (which might be their only element).

Since $Q^{\prime}$ is obtained from $Q$ by a cyclic shift for $j$ places this means that $l(B)+q-p=j$. Since $P^{\prime}$ is obtained from $P$ by a cyclic shift for $i$ places and $i=j$ it follows that $P^{\prime}$ has the following two representations.

(1) $C \underbrace{00 \cdots 00}_{q-p} B$ and

(2) $C B \quad 00 \cdots 00$

$$
\underbrace{}_{q-p}
$$


These two representations cannot exist simultaneously since $C$ is followed once with a 1 and once with a 0 . It follows that the case $i=j$ is impossible.

Step 5. The case where $i \neq j$. Let $D_{1}$ denote the block of $q-p$ successive zeros starting at the $(p+1)$ th place in $Q$. This gives rise to a corresponding block $D_{2}$ in the $(p+1-j) \bmod q$ place in $Q^{\prime}$. (Without loss of generality it can be assumed that $Q^{\prime}$ is obtained by a left cyclic shift of $Q$. The same applies to $P^{\prime}$ and $P$ respectively.) Since both $Q$ and $Q^{\prime}$ start with a 1 and end with $q-p$ successive zeros it follows that $j>q-p$ and $D_{2}$ is therefore confined to the first $p$ places of $Q^{\prime}$. Since the first $p$ elements of $Q^{\prime}$ consist of $P^{\prime}$ this gives rise to a block $D_{3}$ in $P^{\prime}$ with the same location as $D_{2}$ in $Q^{\prime}$ and this gives rise to a block $D_{4}$ in the $[(p+1-j) \bmod q+i] \bmod p$ place in $P$. A block $D_{5}$ therefore exists in the same place in $Q$. Starting the same process all over again, another block $D_{6}$ is obtained in $Q^{\prime}$. If this block is in the $(p+1)$ th place, the process terminates. Otherwise it goes on following the above procedure. Two blocks $D_{s}$ and $D_{t}$ located in the same sequence $Q, Q^{\prime}$, $P$ or $P^{\prime}$ do not overlap or abut because they are all followed by a 1. (The first element of a sequence is considered to follow the last one.) Since $q$ is finite this process must finally terminate by obtaining a block $D_{m}$ in the $(p+1)$ th place in $Q^{\prime}$.

It is obvious that if $D_{u}$ is in $Q, Q^{\prime}, P$ or $P^{\prime}$ for some $u$, then $u=1(\bmod 4), 2(\bmod 4), 0(\bmod 4)$ or $3(\bmod 4)$, respectively.

Postulate. Let $D_{m}$ be the block which terminates the process. Let the blocks $D_{4 V+1}, V=0,1, \cdots,(m-2) / 4$ be deleted from $Q$. Then the remaining sequence consists of repetitions of a sub-sequence $A$, where $A$ repeats itself at least twice.

Proof. Let all the blocks $D_{u}, u=1,2, \cdots, m$ be deleted from their corresponding sequences $Q, Q^{\prime}, P$ and $P^{\prime}$ and let the remainders of the sequences be denoted by $\bar{Q}, \bar{Q}^{\prime}, \bar{P}$ and $\bar{P}^{\prime}$, respectively. If the $t$ th element of an original sequence still remains after the deleting process, let its new location be denoted by $\bar{t}$.

$Q^{\prime}$ is obtained from $Q$ by a left cyclic shift for $j$ places. For every deleted $D_{4 V+1}$ in $Q$ which starts at the $u$ th place, there is a deleted $D_{4 V+2}$ in $Q^{\prime}$ which starts at the $(u-i) \bmod a$ place. It follows that $\bar{Q}^{\prime}$ is obtained from $\bar{Q}$ by shifting $\bar{Q}$ cyclically to the left for $\bar{j}$ places.

$P^{\prime}$ is obtained from $P$ by a left cyclic shift for $i$ places. For every deleted $D_{4 V+3}$ in $P^{\prime}$ which starts at the $u$ th place, there is a deleted $D_{4(V+1)}$ in $P$ which starts at the $(u+i) \bmod p$ place. It follows that $\bar{P}^{\prime}$ is obtained from $\bar{P}$ by shifting $P$ cyclically to the left for $\bar{i}$ places. 
It is also obvious that $\bar{P}=\bar{Q}$ and $\bar{P}^{\prime}=\bar{Q}^{\prime}$ and since $i \neq j$ it follows that $\bar{i} \neq \bar{j}$.

It can be concluded that $\bar{Q}$ equals some cyclic permutation of itself, and it therefore consists of repetitions of a sub-sequence $A$, whose length is $|\bar{i}-\bar{j}|$.

Step 6. The sufficiency of the condition stated in Lemma 1. The length of $\bar{Q}$ is $p-n(q-p)$ for some $n$. There were $n$ blocks $D_{u}$ deleted from $P$ with at least one sub-sequence $A$ between any two such blocks. $P$ also starts and ends with $A$ which means that there are at least $n+1$ sub-sequences $A$ in $\bar{Q}$. Since the $r$ elements of value 1 must be equally shared among the sub-sequences it follows that $P^{\prime}$ and $Q^{\prime}$ cannot exist for $k<p \cdot q$ if $(r, p-n(q-p))<n+1$ for $n=$ $1,2, \cdots,[p /(q-p)]$, unless they are periodic. It was shown in Steps 2 and 3 that this condition together with $(r, p)=1$ are sufficient for preventing $P$ and $Q$ from being periodic.

If $r>p-n(q-p)$ for some $n$, the sequence $P^{\prime}$ cannot exist for $k<p \cdot q$, since the number of elements of value 1 exceeds the number of available places. This means that if $h$ is the smallest integer such that $r>p-h(q-p)$ it is not necessary to stipulate that $(r, p-n(q-p))<$ $n+1$ for $n \geqq h$.

Step 7. The necessity of the condition stated in Lemma 1 . It should be shown that if $(p, r)>1$ or $(r, p-n(q-p)) \geqq n+1$ for any $n$, $1 \leqq n \leqq h-1$, then it is always possible to find $P^{\prime}$ or $Q^{\prime}$ for $k<p \cdot q$.

If $(p, r)>1$ it was shown in Step 2 that $P$ can be periodic and $P^{\prime}$ can be obtained for $k<p$.

If $(r, p-n(q-p))=n+1$ this means that $(r,(n+1) p-n q)=n+1$ and it follows that $(r, n q)=n+1$. Since $(n, n+1)=1$ it follows that $(r, q)=n+1$ and $Q$ is therefore periodic.

If $t_{n} \triangleq(r, p-n(q-p))>n+1$, let $r=b \cdot t_{n}$ and $p-n(q-p)=$ $g \cdot t_{n}$. The values of $n$ are always such that $g \geqq b$. Let $A$ be a sequence of length $g$ starting with a 1 and having b elements of value 1 in it. The rest of its elements (if exist) are zeros. Let $B$ be a sequence constructed by attaching consecutively $t_{n}-n$ sequences $A$, and let $D$ denote a sequence of $q-p$ zeros.

The sequences $Q$ and $Q^{\prime}$ are constructed by attaching consecutively $A, B$ and $D$ in the following way. $Q=B, D, A, D, A, D, \cdots, A, D$; $Q^{\prime}=A, D, A, D, \cdots, A, D, B, D$; where $A$ and $D$ are written $n$ and $n+1$ times, respectively. It is clear that the sequences $P$ and $P^{\prime}$ obtained from $Q$ and $Q^{\prime}$ by dropping the last $D$, are also obtained each from the other by a cyclic shift.

This completes the proof of Lemma 1. 
Step 8. Conclusion. Lemma 1 was identical to the Theorem for the case $t=2$. The following Lemma is identical to the Theorem in its general form.

Lemma 2. Let $B_{i}, i=1,2,3, \cdots, t$, be binary sequences with $r$ elements of value 1 , where $l\left(B_{t}\right) \stackrel{\Delta}{=} m_{l}$. Let $m_{t}>m_{t-1}>\cdots>m_{2}>m_{1}>r$, and $\left(m_{l}, m_{j}\right)=1$ for $i \neq j$. The first $m_{1}$ elements of all $B_{\imath}$ consist of the sequence $B_{1}$, with their first element 1 . Let all $B_{1}$ be shifted cyclically through $k$ places, until the sequences $B_{i}^{\prime}$ are obtained, respectively, where the first $m_{1}$ elements of all sequences consist of the sequence $B_{1}^{\prime}$, their first element being 1 . Let $s_{1 j} \stackrel{\Delta}{=} m_{1}-m_{j}$.

Then $k$ is only of the form $m \cdot \prod_{i=1}^{t} m_{i}$, for some integer $m$, iff $\left(r, m_{i}\right)=1$ and $\left(r, m_{i}-n \cdot s_{i j}\right)<n+1$ for $n=1,2, \cdots, h-1$ where $h$ is the smallest integer such that $r>m_{\imath}-h \cdot s_{i j}$. This applies to $i=$ $1,2,3, \cdots, t-1$ and all $j<i$.

The proof of Lemma 2 follows directly by the application of the Chinese Remainder Theorem to Lemma 1, and the proof of the Theorem is thus complete.

Acknowledgement. The author is thankful to a Referee for making constructive suggestions which enabled a considerable simplification of the proof of the Theorem.

Received August 23, 1976 and in revised form February 8, 1977.

National Electrical Engineering Research Institute

P. O. BOX 395

Pretoria 0001, South Africa 


\section{PACIFIC JOURNAL OF MATHEMATICS \\ EDITORS}

RICHARD ARENS (Managing Editor)

University of California

Los Angeles, CA 90024

\section{R. A. Beaumont \\ University of Washington \\ Seattle, WA 98105}

C. C. MOORE

University of California

Berkeley, CA 94720

\section{J. DUGUNDJI}

Department of Mathematics

University of Southern California

Los Angeles, CA 90007

R. FINN AND J. MILGRAM

Stanford University

Stanford, CA 94305

\section{ASSOCIATE EDITORS}

\section{E. F. BECKENBACH}

B. H. NeUmanN

F. WOLF

K. YoSHIDA

\section{SUPPORTING INSTITUTIONS}

\author{
UNIVERSITY OF BRITISH COLUMBIA \\ CALIFORNIA INSTITUTE OF TECHNOLOGY \\ UNIVERSITY OF CALIFORNIA \\ MONTANA STATE UNIVERSITY \\ UNIVERSITY OF NEVADA \\ NEW MEXICO STATE UNIVERSITY \\ OREGON STATE UNIVERSITY \\ UNIVERSITY OF OREGON \\ OSAKA UNIVERSITY
}

\author{
UNIVERSITY OF SOUTHERN CALIFORNIA \\ STANFORD UNIVERSITY \\ UNIVERSITY OF HAWAII \\ UNIVERSITY OF TOKYO \\ UNIVERSITY OF UTAH \\ WASHINGTON STATE UNIVERSITY \\ UNIVERSITY OF WASHINGTON \\ AMERICAN MATHEMATICAL SOCIETY
}

The Supporting Institutions listed above contribute to the cost of publication of this Journal, but they are not owners or publishers and have no responsibility for its contents or policies.

Mathematical papers intended for publication in the Pacific Journal of Mathematics should be in typed form or offset-reproduced (not dittoed), double spaced with large margins. Underline Greek letters in red, German in green, and script in blue. The first paragraph or two must be capable of being used separately as a synopsis of the entire paper. Items of the bibliography should not be cited there unless absolutely necessary, in which case they must be identified by author and Journal, rather than by item number. Manuscripts, in duplicate, may be sent to any one of the four editors. Please classify according to the scheme of Math. Reviews, Index to Vol. 39. All other communications should be addressed to the managing editor, or Elaine Barth, University of California, Los Angeles, California, 90024.

100 reprints are provided free for each article, only if page charges have been substantially paid. Additional copies may be obtained at cost in multiples of 50 .

The Pacific Journal of Mathematics is issued monthly as of January 1966. Regular subscription rate: $\$ 72.00$ a year (6 Vols., 12 issues). Special rate: $\$ 36.00$ a year to individual members of supporting institutions.

Subscriptions, orders for back numbers, and changes of address should be sent to Pacific Journal of Mathematics, 103 Highland Boulevard, Berkeley, California, 94708.

PUBLISHED BY PACIFIC JOURNAL OF MATHEMATICS, A NON-PROFIT CORPORATION

Printed at Jerusalem Academic Press, POB 2390, Jerusalem, Israel.

Copyright (C) 1977 Pacific Journal of Mathematics All Rights Reserved 


\section{Pacific Journal of Mathematics \\ Vol. 70, No. $2 \quad$ October, 1977}

B. Arazi, A generalization of the Chinese remainder theorem ........... 289

Thomas E. Armstrong, Polyhedrality of infinite dimensional cubes .... . . . 297

Yoav Benyamini, Mary Ellen Rudin and Michael L. Wage, Continuous

images of weakly compact subsets of Banach spaces ............ 309

John Thomas Burns, Curvature functions on Lorentz 2-manifolds ......... 325

Dennis F. De Riggi and Nelson Groh Markley, Shear distality and equicontinuity .................................. 337

Claes Fernström, Rational approximation and the growth of analytic

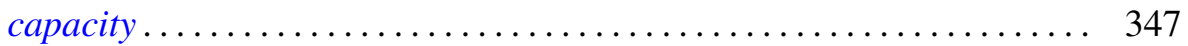

Pál Fischer, On some new generalizations of Shannon's inequality....... 351

Che-Kao Fong, Quasi-affine transforms of subnormal operators ......... 361

Stanley P. Gudder and W. Scruggs, Unbounded representations of

*-algebras........................................ 369

Chen F. King, A note on Drazin inverses .................... 383

Ronald Fred Levy, Countable spaces without points of first countability . . . 391

Eva Lowen-Colebunders, Completeness properties for convergence

spaces ......................................... 401

Calvin Cooper Moore, Square integrable primary representations ....... 413

Stanisław G. Mrówka and Jung-Hsien Tsai, On preservation of

E-compactness ................................ 429

Yoshiomi Nakagami, Essential spectrum $\Gamma(\beta)$ of a dual action on a von

Neumann algebra ................................ 437

L. Alayne Parson, Normal congruence subgroups of the Hecke groups

$G\left(2^{(1 / 2)}\right)$ and $G\left(3^{(1 / 2)}\right)$...

Louis Jackson Ratliff, Jr., On the prime divisors of zero in form rings . . . . 489

Caroline Series, Ergodic actions of product groups .................. 519

Robert O. Stanton, Infinite decomposition bases..................... 549

David A. Stegenga, Sums of invariant subspaces .................. 567 